Copyright $@ 2010$ Institute of Electrical and electronics Engineers, Inc.

All Rights reserved.

Personal use of this material, including one hard copy reproduction, is permitted.

Permission to reprint, republish and/or distribute this material in whole or in part for any other purposes must be obtained from the IEEE.

For information on obtaining permission, send an e-mail message to stds-igr@ieee.org.

By choosing to view this document, you agree to all provisions of the copyright laws protecting it.

Individual documents posted on this site may carry slightly different copyright restrictions.

For specific document information, check the copyright notice at the beginning of each document. 


\title{
The Impact of Cross-functional Teams on Operational Performance after the Implementation of Intelligent Information Systems
}

\author{
Ricardo Santa ${ }^{1}$, W K Daniel Pun ${ }^{2}$ \\ 1 Charles Darwin University, Darwin NT 0909 Australia, Ricardo.Santa@cdu.edu.au \\ 2 CQUniversity Australia, Rockhampton QLD 4702 Australia, D.Pun@cqu.edu.au
}

\begin{abstract}
This study examines cross-functional teams and their impact in line with operational performance and system effectiveness after the implementation of intelligent information and retrieval systems in organisations. Multiple regression analysis was used to determine the structural relationships and look for the phenomenon of cross-functional teams, operational effectiveness, and system effectiveness in the betterment of operational performance. Preliminary findings suggest that the quality and speed from operational effectiveness and the quality of information and quality of the services from systems effectiveness are the two key factors for cross-functional teams to enhance operational performance.
\end{abstract}

Keywords - Intelligent Information Systems, Operational Effectiveness, Systems Effectiveness

\section{INTRODUCTION}

Innovative organisations are those that use innovation to improve their processes or services [1]. These firms are faced with competitive pressures to improve efficiency and productivity through technological implementation [2]. Consequently, organisations that are seeking not only to survive, but to maximise operational effectiveness in an ever-changing environment, need to identify human resource factors, such as senior management support, training, employee empowerment and teamwork, as key elements in the implementation process of technological innovation [3, 4]. Similarly, organisations involved in innovative processes are increasingly using cross-functional teams to enhance their competitiveness [5], and responsiveness to market changes [1].

Despite the recognition of the importance of such factors, the extent to which these cross-functional project teams help organisations to increase the effectiveness of the operations, and to continuously improve after the implementation of technological innovations, has received little attention from researchers [6]. For instance, Harrison and Storey [7] and Ahmad and Schroeder [8] conducted studies to link human resources issues, such as crossfunctional teams, with operations management, and argued that operations management literature tends to either ignore, or pay little attention to, human resources issues, even given the critical role of people in achieving superior performance in the operations of service organisations.
Given the limited empirical studies that specifically investigated cross-functional teams and their impact on the alignment between technological innovation and operational performance in service organisations, this research uses a quantitative approach to investigate the stated issue. This research addressed the question "What is the contribution of cross-functional teams to the continuous improvement of operational performance, after the implementation of technological innovations, such as Intelligent Systems and Information Retrieval Systems (IRS)?” This research analyses the results of a survey of employees in organisations from the electricity distribution, retail sector and higher education sector, and aims to build on the existing literature, further confirming and refining the theoretical framework.

\section{BEHIND THE SCENE}

\section{A. Cross-Functional Teams}

In an ever-changing environment, superior leaders spend time creating the culture to support innovation, knowledge exchange and collaboration with the aim to improve processes or services $[1,9]$. In the quest to reach the improvement of processes or services, organisations implement technological innovations such intelligent systems to improve the performance of their operations. In the implementation process of technological innovations some organisations are increasingly using human resource factors such as cross-functional teams [4], because crossfunctional teams are often understood as the cooperation or collaboration of individuals drawn from various functional areas [5]. This cross-functional cooperation brings ideas, knowledge, expertise and innovation to the project. For instance, Tidd, Bessant \& Pavitt [1] stated that crossfunctional teams have more to offer than individuals in terms of idea generation, flexibility to solutions developed, and a mechanism for bridging boundaries to create innovative solutions within organisations. Likewise, a company with a strong culture of innovation leverages relationships across boundaries and through the extended enterprise, and encourages people to reach further and more faster to gain or spread knowledge $[9,10]$. Cross-functional teams provide a substantial range of ideas, learnings and improvements that can be applied to the organisation [11]. 
Thus, organisations need to use cross-functional teams in the implementation of innovative technologies, such as intelligent systems, with the aim of bringing ideas, learning, knowledge, expertise and innovation into the project. Managers' efforts to create complex networks to connect their people and partners globally, and to empower employees for cross-functional teams to operate efficiently, however, could be seen as challenging if key factors to succeed are not appropriately defined.

According to [12] many factors, such as empowering project teams, establishing project climate, the human resources of the teams, and the establishment of goals, have been suggested in the literature by a number of different researchers when looking at how to achieve successful cross-functional teams. An appropriate project goal is mentioned most often as being associated with success [5, 12]. The establishment of clear and consistent goals provides the members of the cross-functional team with a common boundary or frame of reference, and keeps the teams oriented towards common tasks and outcomes. This establishment of clear and consistent goals in turn promotes a higher level of cross-functional cooperation [5]. In addition, the team leader also may play an enabling role in fostering cooperation among team members, to keep the team focus on its goals [12]. Successful use of crossfunctional teams in the implementation of technological innovations requires the establishment of clear and consistent goals. Additionally, it is important to identify the expectation of stakeholders as they come from different functional areas and diverse cultures, as this diversity could bring miscommunication or misunderstandings to the cross-functional team.

With regard to organisational cultural diversity, Orlikowski and Gash [13] stated that different stakeholders in an organisation have different assumptions, expectations, knowledge and perceptions of technological innovation. Such interpretations of technological innovation, called technological frames [13] or three cultures of management [14], are central to understanding technological development, use, and change in organisations as they influence the way people behave towards technological innovations. These different stakeholders' expectations need to be analysed and taken into account while determining the project's goals and objectives, as they influence the results of the project [15]. Cross-functional project teams are comprised of different stakeholders who bring a wide array of ideas, expectations and needs. Moreover, as the role of cross-functional project teams is important in effective technological implementation and responses of stakeholders such as managers, engineers, and operatorusers vary, care should be taken to minimise conflicts [13, 15]. Therefore, it is important to establish bridges that assist the members from different cultures or backgrounds in the cross-functional team to succeed in the implementation of a common language, so they can define clear and consistent goals.

\section{B. Operational Effectiveness}

In order to face changing market conditions, service firms need learning processes to build the flexible capability to reconfigure and transform their processes. In dynamic and unstable environments firms need to constantly scan their environment and government policies, and develop agile behaviours or competencies, to rapidly accomplish changes [16]. In addition, an increasing number of factors are prompting organisations to seek ways to operate more efficiently and to ensure they have effective operational processes $[17,18]$. This involves the need to deliver value-adding products or services of exceptional quality, on time and at a competitive price. Thus, organisations attempting to meet these objectives need to pay attention to their operational effectiveness, as this is a primary driver of business performance [18].

Operational effectiveness refers to the ability to establish processes, based on core capabilities within the organisations which work well [19]. Operational effectiveness involves improving process performance by leading and controlling the processes within the firm, as well as measuring and improving the processes. A better use of resources through these core processes enables the organisation to eliminate waste, reduce costs, adapt more appropriate technological innovation and therefore perform better than competitors [19].

The five performance dimensions or objectives an organisation seeks to fulfil to attain operational effectiveness are cost, quality, reliability, flexibility and speed [20, 17].

\section{System Effectiveness}

System effectiveness has been described as the extent to which intelligent systems contribute to achieving organisational goals and benefits [22]. Companies deriving the greatest benefits from their systems are those that, from the start, view the importance of the technological innovation, primarily in strategic and organisational terms. These companies stress the importance of operational effectiveness, not the system.

The initial DeLone and McLean's [23] model contained five variables: system quality, information quality, perceived usefulness, user satisfaction, and intelligent system use. In 2003 DeLone and McLean [22] revised their model to include six interrelated dimensions of intelligent systems success, to measure the dependent variable intelligent system effectiveness. It is important to understand key elements of the relationship between system effectiveness and operational effectiveness and to have a real picture of the impact of technological innovation implementation in the improvements on operational performance.

The main purpose of this research is to build on and extend the existing literature and to put forward a theoretical framework that examines the following propositions: 
1) That there is a relationship between cross-functional teams, system effectiveness, operational effectiveness and continuous improvement of operational performance.

2) That a limited number of factors have the potential to explain the alignment between system effectiveness and operational effectiveness.

3) That cross-functional project teams influence the continuous improvement of the operational performance.

\section{ReSEARCh Issues AND Methodology}

This research is undertaken with an exploratory purpose, as the alignment between system effectiveness, operational effectiveness, and cross-functional teams and their influence in the continuous improvement of operational performance in service organisations, has had little academic investigation. According to Sekaran [24] exploratory study is undertaken when there is a lack of understanding of the problem, which leads to an unstructured problem design. Quantitative data was gathered through a self administered questionnaire. The questionnaire was administered to managers, engineers (technologist), and administrative and operational staff as, according to Orlikowski and Gash [13], different actors in an organisation have different assumptions, expectations, knowledge and perceptions of technological innovation. The questionnaire was also administered in organisations from the electricity distribution and retail sector and from the higher education sector, all of which had recently implemented an intelligent system. In the process of constructing measures of key variables and refining the survey instrument, four pilot tests were conducted.

\section{RESULTS}

\section{A. Assessment of Data Validity}

Before operational effectiveness and system effectiveness can be used together as a measure of the alignment between system effectiveness and operational effectiveness, after the implementation of an intelligent system, it is necessary to assess its validity. The questionnaires were emailed to employees in managerial or executive roles, information technology, intelligent systems or engineering roles, and operators or general staff in the selected service organisations. Of the 450 surveys distributed among the service organisations from the electricity distribution, retail sector and higher education sector, 144 were returned (32\% response). Each returned questionnaire was reviewed for completeness and, of the 144, 6 were considered unusable due to large amounts of missing data, lack of involvement of the respondent in the use of intelligent system, or the impossibility of identifying the role of the respondent (manager, engineer or operator-user).

The cross-functional teams section of the questionnaire reported a reasonably high Cronbach's Alpha coefficient

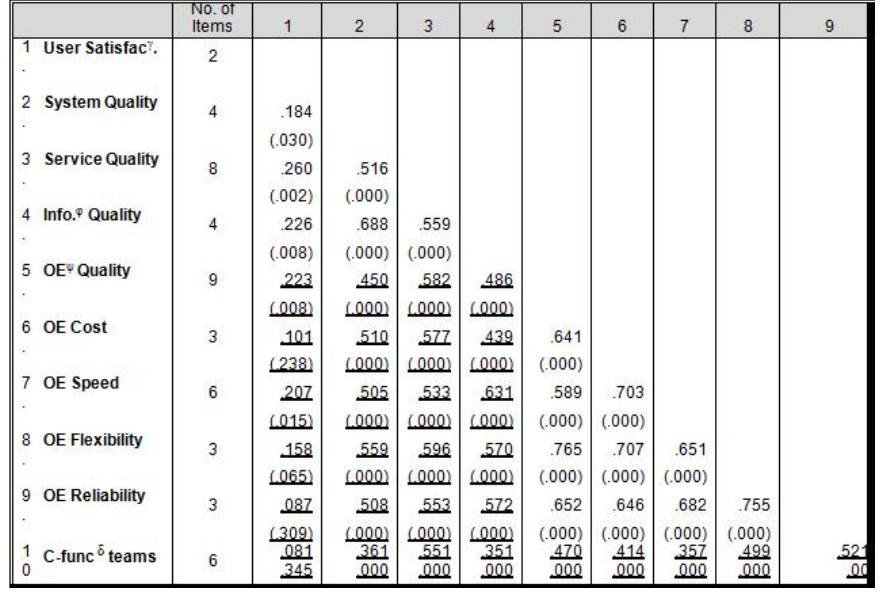

Fig. 1. Reliabilities and pearson correlation matrix.

of 0.743 . The system effectiveness section of the questionnaire reported a Cronbach's Alpha coefficient of 0.859 . The operational effectiveness section reported a Cronbach's Alpha coefficient of 0.936. This high coefficient supported the argument for bringing the dimensions of operational effectiveness into the system effectiveness context to have a more comprehensive understanding of the real effectiveness of the intelligent system implementation. The continuous improvement of operational performance section reported a Cronbach's Alpha coefficient of 0.862 . These Cronbach's Alpha coefficients indicated a high level of internal consistency within these measures as the generally accepted lower limit is 0.7 , though some studies allow 0.6; for example, Hair et al. [25].

The strength and nature of relationships between the dimensions stemming from system effectiveness (system quality, information quality, service quality, user satisfaction) and the performance objectives stemming from operational effectiveness (cost, quality, flexibility speed and reliability), and cross-functional teams, shown as underlined areas in Fig. 1, were investigated using the Pearson product-moment correlation coefficient. There were strong, positive and significant correlations between some variables, such as $r=.631, p<.001$ for Information Quality and Operational Effectiveness (OE) Speed, $r=$ $.596, p<.001$ for Service Quality and Operational Effectiveness Flexibility, $r=.577, p<.001$ for Service Quality and Operational Effectiveness Cost, $r=.572, p<.001$ for Information Quality and Operational Effectiveness Reliability, as shown in the underlined area in Fig. 1. The high correlation and significance means that the performance objectives stemming from operational effectiveness can be used to measure the impact of the implementation of technological innovations such as intelligent systems on operational effectiveness, and the important impact of cross-functional teams in the alignment between system effectiveness and operational effectiveness. 


\section{B. Factor Analysis}

As the main purpose of the study was to explore the alignment between system effectiveness and operational effectiveness and the role played by cross-functional teams in the stated alignment and in the continuous improvement of operational performance, the next step in the data analysis is to perform a factor analysis to reduce the dimensionality and to identify the most important clusters from system effectiveness and operational effectiveness, while at the same time eliminating the items that are less representative. The factor analysis was performed using the maximum likelihood extraction method and oblique rotation method which allows the factors to be correlated. During the factor analysis process, four factors emerged. Hair et al [25] argue that in order to ensure a power level of $80 \%$, a factor loading of 0.55 is significant if the sample size is at least 100 observations at a significant level $(\alpha)$ of 0.05 . Thus, only factor loadings of at least 0.60 have been considered. The solution resulted in a Kaizer-MeyerOlkin (KMO) value of 0.832 with four factors accounting for $52.65 \%$ of the cumulative variance, indicating a satisfactory solution. The four factors are: quality and speed stemming from operational effectiveness, and quality of information and quality of the service stemming from system effectiveness. These four factors also demonstrate the focus that organisations need to consider when aligning technological innovations with operational effectiveness. It is expected also that this alignment will assist organisations to enhance continuous improvement.

\section{Path Analysis}

Forward multiple regression was used to assess the ability of the two dimensions stemming from system effectiveness, the two performance objectives stemming from operational effectiveness, and cross-functional teams to predict continuous improvement of operational performance. Furthermore, path analysis has assisted in describing the entire structure of linkages between independent and dependent variables [26]. Preliminary analysis was conducted to ensure no violation of the assumptions of normality, linearity, muticollinearity and homoscedascity [27, 28].

The results from our coefficients table generated from the regression analysis show that only three dimensions or performance objectives entered the prediction equation as shown in Fig. 2 (Factor-1 'Operational Effectiveness Quality': $\beta=.59$; Factor-3 'System Effectiveness Information Quality': $\beta=.35$; Factor-2 'Operational Effectiveness Speed': $\beta=.14$ ). Quality of the service stemming from system effectiveness and Cross-functional Teams has no predictive power or contribution on Continuous Improvement of Operational Performance. Moreover, the results from the coefficients table show that Crossfunctional Teams is a significant predictor of the two dimensions stemming from system effectiveness and the two performance objectives stemming from operational

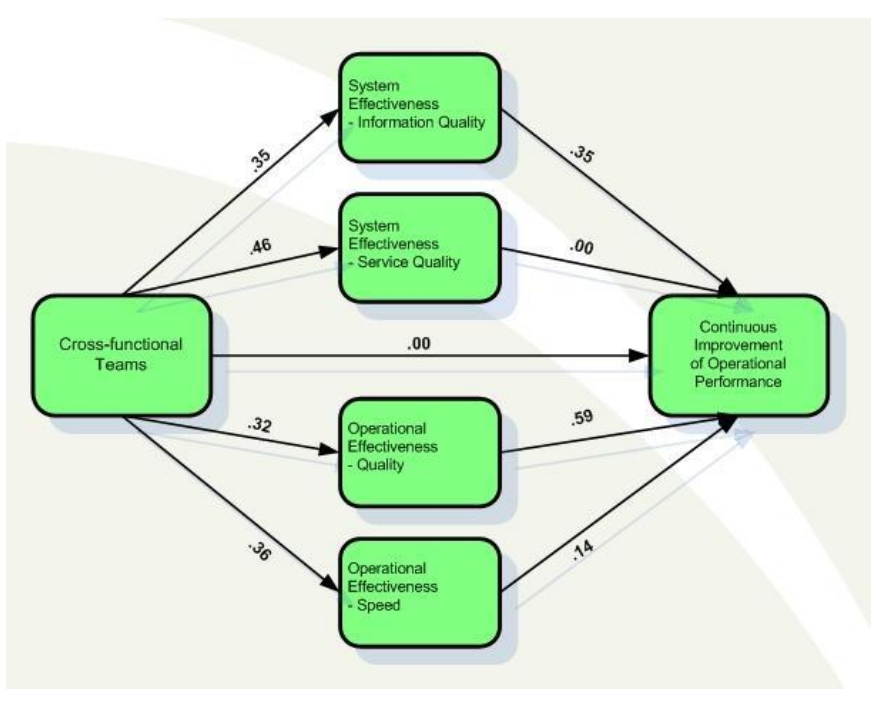

Fig. 2. Research model.

effectiveness (Factor-1 'Operational Effectiveness Quality': $\beta=.32$; Factor-2 'Operational Effectiveness Speed': $\beta=.36$; Factor-3 'System Effectiveness Information Quality': $\beta=.35$; Factor-4 'System Effectiveness Service Quality': $\beta=.46$ ).

Fig. 2 presents the path model together with the estimated regression coefficients (Beta values: $\beta$ ) associated with the hypothesised paths. The path analysis shows direct contribution from Cross-functional Teams on system effectiveness and operational effectiveness; however, there is no direct contribution from Cross-functional Teams on the Continuous Improvement of Operational Performance.

Our results show that the predictors or independent variables for Continuous Improvement of Operational Performance generated a significant prediction equation.

\section{CONCLUSiON}

In answering the research question 'What is the contribution of cross-functional teams to the continuous improvement of operational performance after the implementation of technological innovations, such as intelligent systems?' this study found that there is no direct influence of cross-functional teams on the continuous improvement of operational performance. The lack of direct influence of cross-functional teams on continuous improvement of operational performance can be explained by the fact that improved operational performance is the result of the interaction between cross-functional teams and its related outcomes, i.e. quality of the information, and quality and speed of the operations. Likewise, this study has demonstrated that the cross-functional teams have an important role in both the effectiveness of the operations and the effectiveness of the implemented innovative technology. Consequently, operational performance can be improved by cross-functional teams, but only via the effectiveness 
of operations and the effectiveness of the technological innovation.

It is important to note that decisions about innovation are made based on evidence, so organisations need highquality information. One of the problems in continuously innovating organisations is that although they implement an intelligent system, this does not lead to operational effectiveness. The organisations studied are becoming more complex and more dynamic and they are seeking to innovate to deliver high-quality services, cheaper and faster. The extent to which this innovation helps organisations in the delivery of better services and in reducing operational cost, however, is questioned by this study. Our suggestion is that organisations must work on all the dimensions mentioned in this research, to maximise the probability of achieving cross-functional team success and avoid the conflicts among different technological frames. The challenge for these organisations is to improve the quality of the information, the quality of services and the speed to deliver high-quality services to the end customers, as the research has revealed the importance of these dimensions and performance objectives in achieving the continuous improvement of operational performance.

\section{REFERENCES}

[1] J. Tidd, J. Bessant, J, K. and Pavitt, K, Managing Innovation, USA: John Wiley and Sons, Ltd, 2001.

[2] P. Ifandoudas and R. Chapman, "Strategic ICT implementation within SMEs for business Improvement: The COSTWORTH project", paper presented to 6th International CINet Conference, Brighton, United Kingdom, 2006.

[3] M. Beer and R. A. Eisenstat, "Developing an organisation capable of implementing strategy and learning," Human Relations, vol. 49, no. 5, pp. 597-617, 1996.

[4] B. Daily and S. Huang, "Achieving sustainability through attention to human resource factors in environmental management", International Journal of Operations \& Production Management, vol. 21, no. 12, pp. 1539-1552, 2001.

[5] M. Pinto, J. Pinto, and J. Prescott, "Antecedents and consequences of project team cross-functional cooperation," Management Science, vol. 39, no. 10, pp. 1281-1297, 1993.

[6] M. Koulikoff-Souviron and A. Harrison, "The pervasive human resource picture in interdependent supply relationships," International Journal of Operations \& Production Management, vol. 27, no. 1, pp. 8-27, 2007.

[7] A. Harrison and J. Storey, "New wave manufacturing strategies: operational, organisational and human dimensions," International Journal of Operations \& Production Management, vol. 16, no. 2, pp. 63-76, 1996.
[8] S. Ahmad and R. Schroeder, "The impact of human resource management practices on operational performance: recognising country and industry differences," Journal of Operations Management, vol. 21, pp. 19-43, 2003.

[9] R. Moss, "A Culture of Innovation," Executive Excellence, vol. 17, no. 8, pp. 10-11, 2000.

[10] S. Robbins, Essentials of Organisational Behaviour, Upper Saddle River: San Diego State University, 1997.

[11] H. Boer, S. Caffyn, M. Corso, P. Coughlan, J. Gieskes, M. Magnusson, S. Pavesi, and S. Ronchi, "Knowledge and continuous innovation: the CIMA methodology," International Journal of Operations and Production Management, vol. 21, no. 4, pp. 490504, 2001.

[12] E. McDonough, "Investigation of factors contributing to the success of cross-functional teams," Journal of Production and Innovation Management, vol. 17, no. 3, pp. 221-235, 2000.

[13] W. Orlikowski and D. Gash, "Technological frames: making sense of information technology in organisations," ACM Transactions on Information Systems, vol. 12, no. 2, pp. 174-207, 1994.

[14] E. Schein, "Three cultures of management: the key to organisational learning," Sloan Management Review, vol. 38, no. 1, pp. 9-20, 1996.

[15] A. Elias and O. Zwikael, Stakeholder Participation in Project Management: A New Zealand Study, Melbourne, Australia, 2007.

[16] D. Teece, G. Pisano, and A. Shuen, "Dynamic capabilities and strategic management," Strategic Management Journal, vol. 18, no. 7, pp. 509-533, 1997.

[17] T. Hill, "Strategic Context and Managerial Analysis," Operations Management, Pearson Education Limited, 2000.

[18] N. Slack, S. Chambers, and R. Johnston, Operations Management, 4th edn, Pearson Education Limited, 2004.

[19] M. Porter, "What is strategy?," Harvard Business Review, 1996.

[20] G. White, "A survey and taxonomy of strategy-related performance measures for manufacturing," International Journal of Operations and Production Management, vol. 16, no. 3, pp. 42-61, 1996.

[21] L. M. Corbett, "Delivery windows - a new view on improving manufacturing flexibility and on-time delivery performance," Production and Inventory Management Journal, vol. 33, no. 3, pp. 74-79, 1992.

[22] W. DeLone and E. McLean, "The DeLone and McLean model of information system success: a ten-year update," Journal of Management Information Systems, vol. 19, no. 4, pp. 9-30, 2003.

[23] W. DeLone and E. McLean, "Information systems success: the quest for the dependent variable," Information Systems Research, vol. 3, no. 1, pp. 60-95, 1992.

[24] U. Sekaran, Research Methods for Business, 4th edn, John Wiley and Sons, 2003.

[25] J. F. Hair, R. E. Anderson, R. L. Tatham, and W. C. Black, Multivariate Data Analysis, New Jersey, 1998.

[26] R. Ho, Handbook of Univariate and Multivariate Data Analysis and Interpretation with SPSS, Chapman \& HAll CRC, 2006.

[27] R. Cooksey, Illustrating Statistical Procedures for Business, Behavioural \& Social Science Research, Tilde University Press, 2007.

[28] J. Pallant, SPSS Survival Manual, Crows Nest, NSW, Australia: Allen \& Unwin, 2007. 